\title{
NUEVOS HALLAZGOS DE SITIOS DE CAZADORES RECOLECTORES MARINOS TEMPRANOS EN ISLA ENGLEFIELD, MAR DE OTWAY
}

MANUEL SAN ROMÁN ${ }^{1}$

\section{RESUMEN}

Se presenta información preliminar sobre el hallazgo de dos sitios de cazadores-recolectores marinos en isla Englefield, para los que se han obtenido dataciones radiocarbónicas que los ubican entre el sexto y quinto milenio AP. Las características de la industria ósea recuperada en uno de los yacimientos permiten identificarlo como perteneciente a la tradición cultural Englefield (Emperaire y Laming 1961; Legoupil 1997).

PALABRAS CLAVES: Cazadores-Recolectores marinos, Patagonia meridional, Cultura Englefield, Holoceno medio.

\section{ABSTRACT \\ NEW FINDINGS OF EARLY MARITIME HUNTER-GATHERER SITES IN ENGLEFIELD ISLAND, OTWAY SEA}

Preliminary information about the finding of two maritime hunter-gatherer sites in Englefield Island is presented. Radiocarbon dates that locate them between the sixth and fifth millennium BP have been obtained. The characteristics of the bone industry recovered at one of these sites allow identifying it as part of the Englefield cultural tradition (Emperaire y Laming 1961; Legoupil 1997).

KEY WORDS: maritime hunter-gatherers, southern Patagonia, Englefield culture, middle Holocene.

En el marco de prospecciones orientadas a la búsqueda de la fuente de obsidiana verde (Morello et al. 2004), se detectaron dos nuevos yacimientos arqueológicos en la costa noroccidental de isla Englefield, en la cabecera de una pequeña bahía ubicada hacia el suroeste del casco de la estancia Englefield (Fig. 1), lugar en el que se emplaza el sitio arqueológico epónimo excavado por Joseph Emperaire en 1951 (Emperaire y Laming, 1961). Estos sitios han sido denominados Pizzulic 1 (Pz 1) y Pizzulic 2 ( $\mathrm{Pz} 2)$, y se encuentran emplazados en una antigua terraza marina, a aproximadamente $10 \mathrm{msnm}$, correspondiendo a la misma geoforma en la que se emplazan los sitios de Englefield y Bahía Colorada (cfr. Legoupil 1988).

1 Centro de Estudios del Cuaternario de Fuego-Patagonia (CEQUA), Universidad de Magallanes. Av. Bulnes 01890, Casilla 113-D, Punta Arenas; Chile. manuel.sanroman@umag.cl 


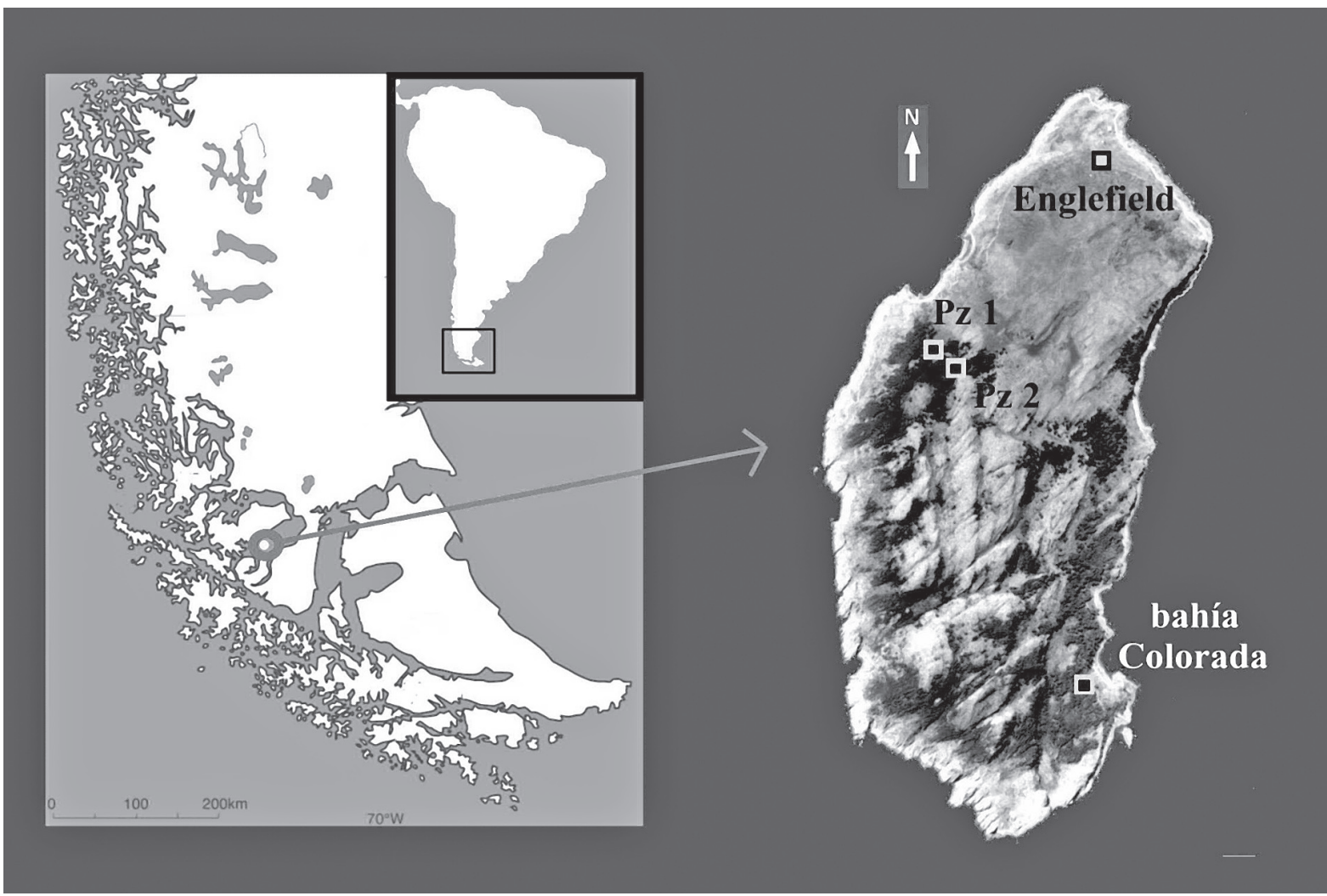

Fig. 1. Mapa con la ubicación de los sitios de canoeros tempranos del estrecho de Magallanes y detalle de la ubicación de los sitios en isla Englefield.

En ambos yacimientos se practicaron excavaciones de sondeo de $1 \mathrm{~m}^{2}$, las que permitieron obtener muestras para realizar dataciones ${ }^{14} \mathrm{C}$. Entre las evidencias recuperadas en ambos sitios destaca la presencia abrumadora de obsidiana verde como materia prima lítica, y la dominancia de restos de fauna marina (Tabla 1). Ambos yacimientos, separados por una quincena de metros, presentan dimensiones dispares: $\mathrm{Pz} 1$ se extiende en un área de aproximadamente $50 \mathrm{~m}^{2}$, y alcanza una potencia estratigráfica máxima de $45 \mathrm{~cm}$. En tanto, el sitio $\mathrm{Pz} 2$ superaría los $250 \mathrm{~m}^{2}$ y alcanza al menos los

Tabla 1. Restos arqueofaunísticos recuperados desde los sondeos (NISP y \%NISP).

\begin{tabular}{|l|c|c|c|c|}
\hline & Pz1 & \%NISP & Pz2 & \%NISP \\
\hline Otaridae & 7 & 0,4 & 270 & 4,39 \\
Delphinidae & 10 & 0,5 & 19 & 0,31 \\
Cetacea & - & - & 10 & 0,16 \\
Lontra sp. & - & - & 1 & 0,02 \\
Arctiodactyla & 2 & 0,1 & 2 & 0,03 \\
Rodentia & 1 & 0,1 & 2 & 0,03 \\
Mammalia & 25 & 1,4 & 175 & 2,84 \\
Aves & 741 & 40,4 & 2054 & 33,36 \\
Peces & 1032 & 56,3 & 3623 & 58,84 \\
Indeterminados & 14 & 0,8 & 1 & 0,02 \\
\hline Total & 1832 & & 6157 & \\
\hline
\end{tabular}




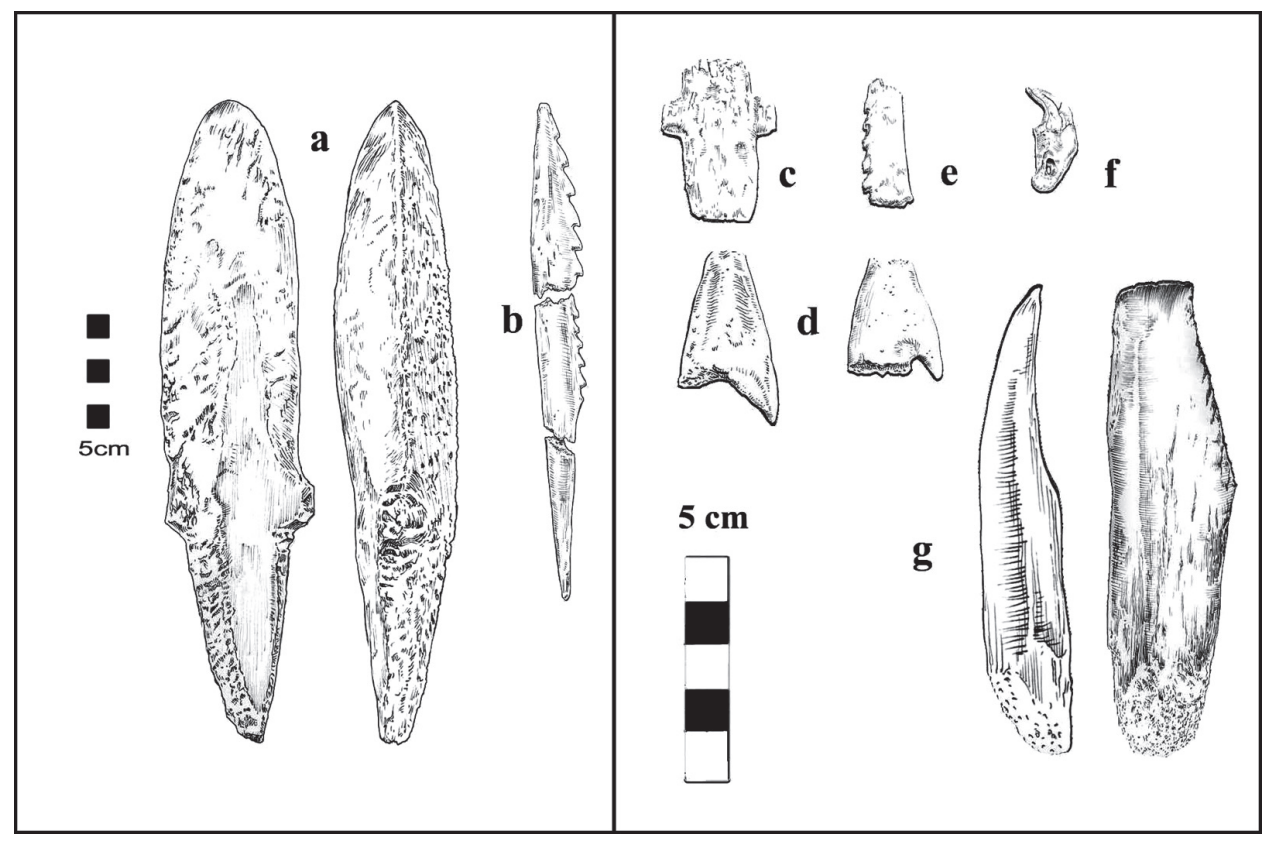

Fig. 2. Instrumentos óseos recuperados desde $\mathrm{Pz} 2$

a) cuña de base cruciforme, b) arpón multidentado, c) base de arpón cruciforme, d) extremos distales de arpones, e) fragmento de arpón multidentado, f) pendiente sobre incisivo de otárido, y g) espátula o cincel sobre ulna de otárido. (dibujos de Nataniel Álvarez).

$65 \mathrm{~cm}$ de potencia ${ }^{1}$. Para este último, se registró una serie de instrumentos óseos característicos de la tradición cultural Englefield (Legoupil, 1997), entre los que destaca un instrumento de gran talla sobre hueso de cetáceo (Fig. 2), semejante a los registrados en el sitio Englefield (cfr. Emperaire y Laming, 1961:22 figura 3, pieza número 6 y 8) y que fueron asignados a la categoría de arpones de base cruciforme (op. cit). En el citado trabajo, se registran únicamente las bases de estos supuestos arpones, aunque en el caso de $\mathrm{Pz} 2$, la pieza recuperada está entera y corresponde a una cuña de base cruciforme.

\section{DATACIONES RADIOCARBÓNICAS}

Se obtuvieron dos dataciones ${ }^{14} \mathrm{C}$ (AMS) para $\mathrm{Pz} 1$, a partir de restos óseos provenientes de la única capa de conchal. La primera de ellas fue realizada sobre Phalacrocorax sp, y arrojó una antigüedad de 6225 \pm 70 años AP (Ua-18929, San Román et al. 2002). La segunda correspondió a un

1 La extensión fue calculada a partir de la distribución de la matriz conchífera. fragmento de metapodio de Arctiodactyla fechada en $5595 \pm 45$ años AP (Ua-21032). La procedencia estratigráfica permite plantear que las diferencias en los resultados pueden ser explicadas - en parte - por el envejecimiento provocado por efecto reservorio sobre los restos de cormorán, anotando que la capa de conchal de $\mathrm{Pz} 1$ es poco espesa, y sugiere un tiempo de formación corto.

Para $\mathrm{Pz} 2$ se obtuvieron dos fechados AMS sobre carbón de fogones superpuestos estratigráficamente. La primera muestra proviene del techo de la segunda capa de conchal (Fig. 3) y la segunda corresponde a un lente próximo a la base de la misma capa. Los resultados obtenidos fueron $5945 \pm 45$ años AP (Ua-21030) y 6055 \pm 60 años AP (Ua-21031), respectivamente. Estos resultados permiten establecer que la formación de la segunda capa del conchero habría ocurrido en un lapso relativamente corto, del orden de los 110 años radiocarbónicos, y permiten postular que la segunda capa de conchero habría sido originada por la reutilización de un mismo locus de habitación, evidenciada a partir de la exacta superposición de dos estructuras de combustión en un lapso de al menos un siglo. 


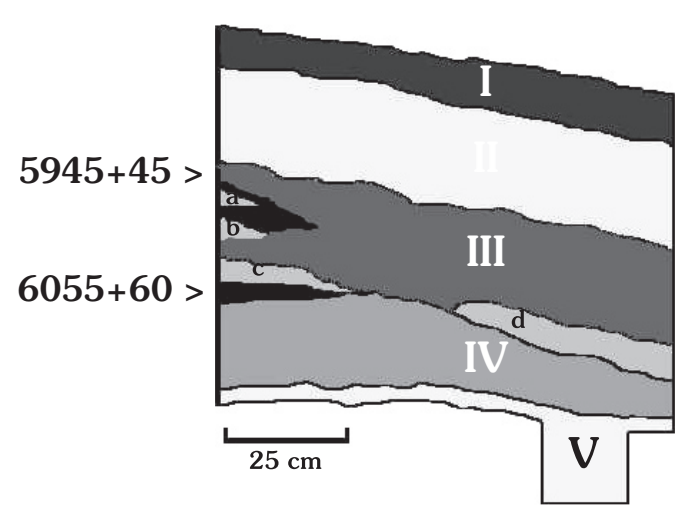

Fig. 3. Esquema de la estratigrafía de $\mathrm{Pz} 2$, perfil oeste. I) Humus, II) Conchal I, de color violáceo claro, III) Conchal II, de color oscuro, IV) Limo y conchas, V) Arcilla compacta, arqueológicamente estéril. Lentes a, b, c y d corresponden a conchilla calcinada y cenizas, de color amarillento. Los lentes de color negro corresponden a carbón vegetal de fogones.

\section{COMENTARIOS FINALES}

El emplazamiento y los resultados de las dataciones ${ }^{14} \mathrm{C}$ de los yacimientos $\mathrm{Pz} 1$ y $\mathrm{Pz} 2$ permiten confirmar que la edad mínima de las terrazas de 10 msnm en la cuenca del mar de Otway alcanza los 6100 años AP. Sin embargo, deberá profundizarse el estudio estratigráfico de la base de los depósitos arqueológicos y de depósitos naturales, orientados a detectar paleoplayas que permitan datar de manera directa la formación de estas geoformas. En este sentido, ya Emperaire sugirió que hacia la base del depósito estratigráfico del sitio Englefield (Emperaire y Laming, 1961:15) habría acumulación de conchillas y rodados que podrían interpretarse como antiguas barras de tormenta, o bermas de altas mareas. La evidencia arqueológica de sitios tempranos de canoeros ha demostrado que el emplazamiento de yacimientos en la cuenca del Otway y estrecho de Magallanes está condicionada a los bordes costeros de la época, y que los criterios de búsqueda de yacimientos de este período deben guiarse por la búsqueda de evidencias en estas antiguas líneas costeras, ubicadas actualmente por sobre los $10 \mathrm{msnm}$.

Por otro lado, las fechas obtenidas para los sitios tempranos de isla Englefield muestran que el lapso en el que fue utilizada la antigua terraza de la transgresión marina Flandrian se ubica con exclusividad entre 6100 y 5000 años AP. La ausen- cia de fechas posteriores a 5000 años AP puede responder a que cambiarían los emplazamientos de los sitios de campamento luego del descenso del nivel del mar. En este sentido, dataciones obtenidas en sitios como Camden y KM 44 (San Román y Prieto, 2004), muestran que hacia el 3000 AP, se habría utilizado el borde costero disponible hacia esa época, emplazado ahora en terrazas marinas ubicadas a $5 \mathrm{msnm}$ actual. En síntesis, la evidencia actual referida a cronología de ocupación humana de distintas terrazas marinas del sistema mar de Otway - estrecho de Magallanes demostraría que los emplazamientos de yacimientos de cazadores marítimos se ordenan secuencialmente en distintos sistemas de terrazas marinas. Resta aún afinar la cronología de estas geoformas, como también, ubicar las líneas costeras previas a la transgresión marina del Holoceno medio (Flandrian). Esto último permitirá guiar las futuras búsquedas de evidencia de ocupación humana costera anterior a la de los cazadores marítimos de la tradición Englefield, instalados hacia el 6000 AP en la región.

\section{BIBLIOGRAFÍA}

EMPERAIRE, J. y A. LAMING. 1961. Les Gisements Archeólogiques des Iles Englefield et Vivian dans la Mer d'Otway. Journal de la Société des Américanistes 50:7-75.

LEGOUPIL, D. 1988 Ultimas consideraciones sobre las dataciones del sitio de Isla Englefield (seno Otway). Anales del Instituto de la Patagonia, Serie Ciencias Sociales 18:95-98.

1997. Bahía Colorada (Île Englenfield). Recherche sur les Civilisations, Paris.

MORELLO, F.; M. SAN ROMÁN y A. PRIETO. 2004. Obsidiana verde en Fuego-Patagonia: distribución y estrategias tecnológicas. En: Contra Viento y Marea. Arqueología de Patagonia. Compilado por M. T. Civalero, P. Fernández y A. G. Guraieb. Edición del Instituto Nacional de Antropología y Pensamiento Latinoamericano y la Sociedad Argentina de Antropología. Pp. 149-166. Buenos Aires.

SAN ROMÁN, M; F. MORELLO y A. PRIETO. 2002. Nuevos antecedentes sobre la explotación de recursos faunísticos en el mar de Otway y canales adyacentes. Anales del Instituto de la Patagonia, Serie Ciencias Humanas 30:147-154.

SAN ROMÁN, M. y A. PRIETO. 2004. (Dis)continuidad del uso de la obsidiana verde entre poblaciones de adaptación marítima del mar de Otway y estrecho de Magallanes. En: Contra Viento y Marea. Arqueología de Patagonia. Compilado por M. T. Civalero, P. Fernández y A. G. Guraieb. Edición del Instituto Nacional de Antropología y Pensamiento Latinoamericano y la Sociedad Argentina de Antropología. Pp. 571-580. Buenos Aires. 